\title{
optica
}

\section{Experimental detection of polarization-frequency quantum correlations in a photonic quantum channel by local operations: supplementary material}

JiAn-Shun TANG ${ }^{1,2}$, YI-TAO WANG ${ }^{1,2}$, Geng Chen ${ }^{1,2}$, YANG ZOU ${ }^{1,2}$, Chuan-Feng Li ${ }^{1,2+}$, Guang-Can Guo ${ }^{1,2}$, Ying Yu ${ }^{2,3}$, Mi-Feng LI ${ }^{2,3}$, Guo-Wei

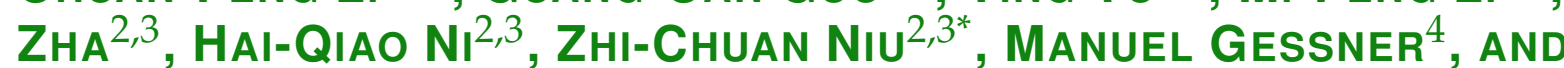
HeINZ-PETER BREUER $4 \S$

${ }^{1}$ Key Laboratory of Quantum Information, University of Science and Technology of China, CAS, Hefei, Anhui 230026, China

${ }^{2}$ Synergetic Innovation Center of Quantum Information \& Quantum Physics, University of Science and Technology of China, Hefei, Anhui 230026, China

${ }^{3}$ The state key laboratory for superlattices and microstructures, Institute of semiconductors, CAS, PO Box 912 , Beijing 100083

${ }^{4}$ Physikalisches Institut, Universität Freiburg, Hermann-Herder-Straße 3, D-79104 Freiburg, Germany

${ }^{\dagger}$ Corresponding author: cfli@ustc.edu.cn

*Corresponding author: zcniu@semi.ac.cn

§Corresponding author: breuer@physik.uni-freiburg.de

Published 30 November 2015

This document provides supplementary information to "Experimental detection of polarization-frequency quantum correlations in a photonic quantum channel by local operations," http://dx.doi.org/10.1364/optica/2.001014. (C) 2015 Optical Society of America

http://dx.doi.org/10.1364/optica/2.001014.s001

\section{CONTENTS}

1 Preparation of the initial state 1

A The pre-initial state . . . . . . . . . . . 1

B The correlated initial state ......... 2

2 The local dephasing operation 2

A Experimental implementation .......... 2

B Reduced states . . . . . . . . . . . 3

C Total trace distance . . . . . . . . . . . . 3

3 Open-system evolution depending on initial correlations

A Local trace distance . . . . . . . . . . . . 4

\section{PREPARATION OF THE INITIAL STATE}

A. The pre-initial state

The pre-initial state is a product state, $\rho_{\mathrm{pi}}=\rho_{0} \otimes \rho_{E}$, where the density matrix of the two-level system represented in the
$\{|H\rangle,|V\rangle\}$ basis reads

$$
\rho_{0}=\left(\begin{array}{cc}
1 / 2 & d e^{i \varphi} \\
d e^{-i \varphi} & 1 / 2
\end{array}\right)
$$

and the environmental state is a mixed state given by a sum over closely spaced frequencies,

$$
\rho_{E}=\sum_{\omega} \Delta \omega G(\omega)|\omega\rangle\langle\omega|
$$

In order to avoid problems with non-normalizable continuum states, we formally represent the state $\rho_{E}$ by a discrete sum of frequencies, and perform the continuum limit $(\Delta \omega \rightarrow 0)$ after obtaining the relevant quantities, such as the trace distance. The frequency states $|\omega\rangle$ form a complete orthonormal set, i.e., $\left\langle\omega \mid \omega^{\prime}\right\rangle=\delta_{\omega, \omega^{\prime}}$ and $\sum_{\omega}|\omega\rangle\langle\omega|=\mathbb{I}$. The conditions $\Delta \omega G(\omega) \geq 0$ and $\sum_{\omega} \Delta \omega G(\omega)=1$ then ensure that $\rho_{E}$ indeed describes a positive and normalized quantum state. In the following we assume for simplicity that $G(\omega)$ is a unimodal function with a width of order $\delta \omega$ which is symmetric about the center frequency $\omega_{0}$. 


\section{B. The correlated initial state}

In order to prepare a quantum state with polarizationfrequency correlations, we send the pre-initial state

$$
\begin{gathered}
\rho_{\mathrm{pi}}=\sum_{\omega} \Delta \omega G(\omega)\left(\frac{1}{2}|H, \omega\rangle\left\langle H, \omega\left|+d e^{i \varphi}\right| H, \omega\right\rangle\langle V, \omega|\right. \\
\left.+d e^{-i \varphi}|V, \omega\rangle\left\langle H, \omega\left|+\frac{1}{2}\right| V, \omega\right\rangle\langle V, \omega|\right)
\end{gathered}
$$

through a birefringent crystal, which generates an interaction of the form

$$
U_{\text {cal }}(t):\left\{\begin{array}{l}
|H, \omega\rangle \rightarrow|H, \omega\rangle \\
|V, \omega\rangle \rightarrow e^{-i \omega t}|V, \omega\rangle,
\end{array},\right.
$$

where $t=\Delta_{\text {cal }} l / c$ corresponds to the time spent inside the crystal. The state $\rho_{\text {pi }}$ evolves into the initial state

$$
\begin{aligned}
& \rho_{S E} \\
& =U_{\mathrm{cal}}(t) \rho_{\mathrm{pi}} U_{\mathrm{cal}}^{+}(t) \\
& =\sum_{\omega} \Delta \omega G(\omega)\left(\frac{1}{2}|H, \omega\rangle\left\langle H, \omega\left|+d e^{i(\omega t+\varphi)}\right| H, \omega\right\rangle\langle V, \omega|\right. \\
& \left.\quad+d e^{-i(\omega t+\varphi)}|V, \omega\rangle\left\langle H, \omega\left|+\frac{1}{2}\right| V, \omega\right\rangle\langle V, \omega|\right) .
\end{aligned}
$$

Its reduced state for the two-level system is given by

$$
\rho_{S}=\left(\begin{array}{cc}
1 / 2 & d C(t) e^{i \Psi(t)} \\
d C(t) e^{-i \Psi(t)} & 1 / 2
\end{array}\right),
$$

with $\Psi(t)=\omega_{0} t+\varphi$ and $\omega_{0}$ denotes the center frequency of the distribution $G(\omega)$. For fixed $t$, the initial phase $\varphi$ can be chosen such that $\Psi(t) \equiv 0$, i.e., $\varphi=-\omega_{0} t$. Since $G(\omega)$ is symmetric about $\omega=\omega_{0}$, we obtain a real-valued function

$$
C(t)=\sum_{\omega} \Delta \omega G(\omega) e^{i\left(\omega-\omega_{0}\right) t}
$$

For the implementation of the local detection protocol, the first step consists in obtaining the eigenbasis of $\rho_{S}$. Diagonalization of this $2 \times 2$ matrix yields the eigenvalues,

$$
\begin{aligned}
& \lambda_{0}=1 / 2+d C(t), \\
& \lambda_{1}=1 / 2-d C(t),
\end{aligned}
$$

and corresponding eigenvectors,

$$
\begin{aligned}
& |0\rangle=\frac{1}{\sqrt{2}}\left(\begin{array}{c}
1 \\
1
\end{array}\right)=\frac{1}{\sqrt{2}}(|H\rangle+|V\rangle), \\
& |1\rangle=\frac{1}{\sqrt{2}}\left(\begin{array}{c}
1 \\
-1
\end{array}\right)=\frac{1}{\sqrt{2}}(|H\rangle-|V\rangle) .
\end{aligned}
$$

Before passing the state on to Bob, Alice implements a random local unitary rotation $U_{r}$ to hide her local eigenbasis. This is implemented by a half-wave plate which is controlled by a random number generator. As a consequence, the eigenstates are rotated to $|\theta\rangle=U_{r}|0\rangle$ and $\left|\theta_{\perp}\right\rangle=U_{r}|1\rangle$. This local unitary operation has no effect on the correlation properties of the state. Redefining the basis vectors $|H\rangle$ and $|V\rangle$ accordingly always allows us to map the state back onto the original non-rotated state. Thus, for the following theoretical analysis, we can restrict to the case $U_{r}=\mathbb{I}$, i.e., effectively disregard this additional local rotation.

\section{THE LOCAL DEPHASING OPERATION}

The local dephasing must be carried out in the eigenbasis of the reduced state (S6). Hence, the desired operation is

$$
\Phi(X)=|0\rangle\langle 0|X| 0\rangle\langle 0|+| 1\rangle\langle 1|X| 1\rangle\langle 1| .
$$

Local application to the state $\rho_{S E}$ yields

$$
\begin{aligned}
\rho_{S E}^{\prime}= & (\Phi \otimes \mathbb{I}) \rho_{S E} \\
= & \frac{1}{2} \sum_{\omega} \Delta \omega G(\omega)[|H, \omega\rangle\langle H, \omega|+| V, \omega\rangle\langle V, \omega| \\
& +d\left(e^{i\left(\omega-\omega_{0}\right) t}+e^{-i\left(\omega-\omega_{0}\right) t}\right)|H, \omega\rangle\langle V, \omega| \\
& \left.+d\left(e^{i\left(\omega-\omega_{0}\right) t}+e^{-i\left(\omega-\omega_{0}\right) t}\right)|V, \omega\rangle\langle H, \omega|\right],
\end{aligned}
$$

where we have used Eq. (S9).

\section{A. Experimental implementation}

We implement the controlled dephasing operation by sending the quantum state through a very long polarizationmaintaining fiber after rotating the eigenstates $|0\rangle$ and $|1\rangle$ onto the principal axes of the fiber with a computer-controlled halfwave plate. Formally, the generated evolution is then described by

$$
U_{\text {deph }}:\left\{\begin{array}{l}
|0, \omega\rangle \rightarrow|0, \omega\rangle \\
|1, \omega\rangle \rightarrow e^{-i \omega s}|1, \omega\rangle
\end{array},\right.
$$

where $s=\Delta_{\mathrm{pmf}} l / c \gg 1 / \delta \omega$ corresponds to the time the photon spends inside the fiber. This can be reformulated as

$$
U_{\text {deph }}:\left\{\begin{array}{l}
|H, \omega\rangle \rightarrow a|H, \omega\rangle+b|V, \omega\rangle \\
|V, \omega\rangle \rightarrow b|H, \omega\rangle+a|V, \omega\rangle
\end{array}\right.
$$

with

$$
a=\frac{1}{2}\left(1+e^{-i \omega s}\right), \quad b=\frac{1}{2}\left(1-e^{-i \omega s}\right) .
$$

We thus obtain

$$
\begin{aligned}
& \rho_{S E}^{\prime \prime} \\
= & U_{\mathrm{deph}} \rho_{S E} U_{\mathrm{deph}}^{\dagger} \\
= & \sum_{\omega} \Delta \omega G(\omega)\left[\frac{1}{2}|H, \omega\rangle\left\langle H, \omega\left|+\frac{1}{2}\right| V, \omega\right\rangle\langle V, \omega|\right. \\
& +d\left(e^{i\left(\omega-\omega_{0}\right) t} a b^{*}+e^{-i\left(\omega-\omega_{0}\right) t} a^{*} b\right)|H, \omega\rangle\langle H, \omega| \\
& +d\left(e^{i\left(\omega-\omega_{0}\right) t} a^{*} b+e^{-i\left(\omega-\omega_{0}\right) t} a b^{*}\right)|V, \omega\rangle\langle V, \omega| \\
& +d\left(e^{i\left(\omega-\omega_{0}\right) t}|a|^{2}+e^{-i\left(\omega-\omega_{0}\right) t}|b|^{2}\right)|H, \omega\rangle\langle V, \omega| \\
& \left.+d\left(e^{i\left(\omega-\omega_{0}\right) t}|b|^{2}+e^{-i\left(\omega-\omega_{0}\right) t}|a|^{2}\right)|V, \omega\rangle\langle H, \omega|\right] .
\end{aligned}
$$

This state realizes the desired dephased state $\rho_{S E}^{\prime}$ to a good approximation. To see this, consider for instance,

$$
|a|^{2}=\frac{1}{2}(1+\cos \omega s) \text {. }
$$

Due to the condition $\delta \omega \cdot s \gg 1$, the function $f(\omega)=\cos \omega s$ oscillates very rapidly compared to variations of $G(\omega)$ and the remaining exponential functions. Such rapid oscillations cannot be resolved and, hence, $|a|^{2}$ must be replaced by its mean value over one period (in frequency space):

$$
|a|^{2} \rightarrow\left\langle|a|^{2}\right\rangle=\frac{1}{2}
$$


We obtain correspondingly

$$
|b|^{2} \rightarrow\left\langle|b|^{2}\right\rangle=\frac{1}{2}, \quad a b^{*} \rightarrow\left\langle a b^{*}\right\rangle=0
$$

With these replacements $\rho_{S E}^{\prime \prime}$ reduces to $\rho_{S E}^{\prime}$.

\section{B. Reduced states}

The reduced states of $\rho_{S E}$ and $\rho_{S E}^{\prime}$ coincide by construction, i.e., $\operatorname{Tr}_{E} \rho_{S E}=\operatorname{Tr}_{E} \rho_{S E}^{\prime}$ and $\operatorname{Tr}_{S} \rho_{S E}=\operatorname{Tr}_{S} \rho_{S E}^{\prime}$. This can be confirmed explicitly as follows. First note that

$$
\begin{aligned}
& \rho_{S E}-\rho_{S E}^{\prime} \\
= & \sum_{\omega} \Delta \omega G(\omega) f(\omega)[|H, \omega\rangle\langle V, \omega|-| V, \omega\rangle\langle H, \omega|],
\end{aligned}
$$

where

$$
f(\omega)=\frac{d}{2}\left(e^{i\left(\omega-\omega_{0}\right) t}-e^{-i\left(\omega-\omega_{0}\right) t}\right)=i d \sin \left[\left(\omega-\omega_{0}\right) t\right]
$$

is purely imaginary and anti-symmetric about $\omega=\omega_{0}$. One immediately confirms that

$$
\rho_{E}-\rho_{E}^{\prime}=\operatorname{Tr}_{S}\left\{\rho_{S E}-\rho_{S E}^{\prime}\right\}=0
$$

Tracing over the environmental degrees of freedom thus yields

$$
\begin{aligned}
& \rho_{S}-\rho_{S}^{\prime} \\
= & \operatorname{Tr}_{E}\left\{\rho_{S E}-\rho_{S E}^{\prime}\right\} \\
= & \sum_{\omega} \Delta \omega G(\omega) f(\omega)[|H\rangle\langle V|-| V\rangle\langle H|] .
\end{aligned}
$$

We now perform the continuum limit $\Delta \omega \rightarrow 0$, leading to

$$
\sum_{\omega} \Delta \omega G(\omega) f(\omega) \longrightarrow \int d \omega G(\omega) f(\omega)=0
$$

In the final step, we have used that $G(\omega)$ is a symmetric function about $\omega=\omega_{0}$, while $f(\omega)$ is anti-symmetric. Hence, we confirm

$$
\rho_{S}-\rho_{S}^{\prime}=0
$$

\section{Total trace distance}

The difference between the two states $\rho_{S E}$ and $\rho_{S E}^{\prime}$ is given by

$$
\begin{aligned}
X= & \rho_{S E}-\rho_{S E}^{\prime} \\
=\frac{1}{2} \sum_{\omega} \Delta \omega & G(\omega) d\left(e^{i\left(\omega-\omega_{0}\right) t}-e^{-i\left(\omega-\omega_{0}\right) t}\right) \\
& \times(|H, \omega\rangle\langle V, \omega|-| V, \omega\rangle\langle H, \omega|) .
\end{aligned}
$$

Diagonalization yields eigenvalues

$$
\mu_{ \pm}(\omega)= \pm i \Delta \omega G(\omega) f(\omega)
$$

and eigenstates

$$
\left|\mu_{ \pm}(\omega)\right\rangle=\frac{1}{\sqrt{2}}(|H\rangle \pm i|V\rangle) \otimes|\omega\rangle
$$

The trace norm of $X$, which corresponds to the trace distance of $\rho_{S E}$ and $\rho_{S E}^{\prime}$ can thus be written as

$$
\begin{aligned}
\delta\left(\rho_{S E}\right) & =\left\|\rho_{S E}-\rho_{S E}^{\prime}\right\| \\
& =\operatorname{Tr} \sqrt{X^{\dagger} X} \\
& =\sum_{\omega}\left(\left|\mu_{-}(\omega)\right|+\left|\mu_{+}(\omega)\right|\right) \\
& =2 \sum_{\omega}|\Delta \omega G(\omega) f(\omega)| \\
& =2 \sum_{\omega} \Delta \omega G(\omega)|f(\omega)| \\
& =d \sum_{\omega} \Delta \omega G(\omega)\left|e^{i\left(\omega-\omega_{0}\right) t}-e^{-i\left(\omega-\omega_{0}\right) t}\right| .
\end{aligned}
$$

Performing the continuum limit finally leads to

$$
\delta\left(\rho_{S E}\right)=d \int d \omega G(\omega)\left|e^{i\left(\omega-\omega_{0}\right) t}-e^{-i\left(\omega-\omega_{0}\right) t}\right|
$$

which for the parameter used in the experiment $(d=1 / 2)$ yields the result given in Eq. (7) of the main manuscript.

\section{OPEN-SYSTEM EVOLUTION DEPENDING ON INITIAL CORRELATIONS}

For the dynamical local detection of the correlations in $\rho_{S E}$, we subject both $\rho_{S E}$ and $\rho_{S E}^{\prime}$ to the Michelson delay setup (see main manuscript), generating the dynamics

$$
U(\eta, \tau):\left\{\begin{array}{ll}
|\eta, \omega\rangle & \longrightarrow|\eta, \omega\rangle \\
\left|\eta_{\perp}, \omega\right\rangle & \longrightarrow e^{-i \omega \tau}\left|\eta_{\perp}, \omega\right\rangle
\end{array},\right.
$$

where $\tau=2 x / c$ correponds to the coordinate of the delayable mirror (M2) and the half-wave plate (HWP2) is rotated to the angle $\eta / 2$, generating the basis vectors

$$
\begin{aligned}
|\eta\rangle & =\left(\begin{array}{c}
\cos \eta \\
\sin \eta
\end{array}\right), \\
\left|\eta_{\perp}\right\rangle & =\left(\begin{array}{c}
-\sin \eta \\
\cos \eta
\end{array}\right) .
\end{aligned}
$$

The original polarization vectors $|H\rangle$ and $|V\rangle$ can be expressed in terms of the new basis vectors $|\eta\rangle$ and $\left|\eta_{\perp}\right\rangle$ as

$$
\begin{gathered}
|H\rangle=\cos \eta|\eta\rangle-\sin \eta\left|\eta_{\perp}\right\rangle, \\
|V\rangle=\sin \eta|\eta\rangle+\cos \eta\left|\eta_{\perp}\right\rangle .
\end{gathered}
$$

(S32)

Hence, the evolution of the original states is given by

$$
U(\eta, \tau):\left\{\begin{array}{l}
|H, \omega\rangle \longrightarrow \cos \eta|\eta, \omega\rangle-\sin \eta e^{-i \omega \tau}\left|\eta_{\perp}, \omega\right\rangle \\
|V, \omega\rangle \longrightarrow \sin \eta|\eta, \omega\rangle+\cos \eta e^{-i \omega \tau}\left|\eta_{\perp}, \omega\right\rangle
\end{array}\right.
$$




\section{A. Local trace distance}

The operator $X=\rho_{S E}-\rho_{S E}^{\prime}$ evolves under this dynamics as

$$
\begin{aligned}
& U(\eta, \tau)\left(\rho_{S E}-\rho_{S E}^{\prime}\right) U^{\dagger}(\eta, \tau) \\
= & \sum_{\omega} \Delta \omega G(\omega) f(\omega) \\
& \times\left[\sin \eta \cos \eta|\eta, \omega\rangle\left\langle\eta, \omega\left|+\cos ^{2} \eta e^{i \omega \tau}\right| \eta, \omega\right\rangle\left\langle\eta_{\perp}, \omega\right|\right. \\
& \quad-\sin ^{2} \eta e^{-i \omega \tau}\left|\eta_{\perp}, \omega\right\rangle\left\langle\eta, \omega|-\sin \eta \cos \eta| \eta_{\perp}, \omega\right\rangle\left\langle\eta_{\perp}, \omega\right| \\
& \quad-\text { H.c. }] \\
= & \sum_{\omega} \Delta \omega G(\omega) f(\omega) \\
& \times\left[e^{i \omega \tau}|\eta, \omega\rangle\left\langle\eta_{\perp}, \omega\left|-e^{-i \omega \tau}\right| \eta_{\perp}, \omega\right\rangle\langle\eta, \omega|\right] .
\end{aligned}
$$

Since we only observe the dynamics of the polarization states, we take the partial trace, which yields

$$
\begin{aligned}
& \operatorname{Tr}_{E}\left\{U(\eta, \tau)\left(\rho_{S E}-\rho_{S E}^{\prime}\right) U^{\dagger}(\eta, \tau)\right\} \\
= & \sum_{\omega} \Delta \omega G(\omega) f(\omega)\left[e^{i \omega \tau}|\eta\rangle\left\langle\eta_{\perp}\left|-e^{-i \omega \tau}\right| \eta_{\perp}\right\rangle\langle\eta|\right] .
\end{aligned}
$$

Represented as a matrix in the basis $\left\{|\eta\rangle,\left|\eta_{\perp}\right\rangle\right\}$, this reads

$$
\operatorname{Tr}_{E}\left\{U(\eta, \tau)\left(\rho_{S E}-\rho_{S E}^{\prime}\right) U^{\dagger}(\eta, \tau)\right\}=\left(\begin{array}{cc}
0 & z \\
z^{*} & 0
\end{array}\right),
$$

where

$$
z=\sum_{\omega} \Delta \omega G(\omega) f(\omega) e^{i \omega \tau}
$$

Note that this result is independent of $\eta$. The trace norm of the operator (S36) corresponds to the trace distance of the locally accessible polarization states $\rho_{S}(\tau)$ and $\rho_{S}^{\prime}(\tau)$ and can be determined as

$$
\begin{aligned}
\Delta(\tau) & =\left\|\rho_{S}(\tau)-\rho_{S}^{\prime}(\tau)\right\| \\
& =2|z| \\
& =2\left|\sum_{\omega} \Delta \omega G(\omega) f(\omega) e^{i \omega \tau}\right| \\
& =d\left|\sum_{\omega} \Delta \omega G(\omega)\left(e^{i\left(\omega-\omega_{0}\right) t}-e^{-i\left(\omega-\omega_{0}\right) t}\right) e^{i \omega \tau}\right| .
\end{aligned}
$$

The continuum limit then yields

$$
\Delta(\tau)=d\left|\int d \omega G(\omega)\left(e^{i\left(\omega-\omega_{0}\right) t}-e^{-i\left(\omega-\omega_{0}\right) t}\right) e^{i \omega \tau}\right| .
$$

By comparing this result to Eq. (S29), one readily confirms that the general inequality

$$
\left\|\operatorname{Tr}_{E}\left\{U(\eta, \tau)\left(\rho_{S E}-\rho_{S E}^{\prime}\right) U^{\dagger}(\eta, \tau)\right\}\right\| \leq\left\|\rho_{S E}-\rho_{S E}^{\prime}\right\|
$$

here is satisfied as a consequence of the triangular inequality.

In the present experiment, the frequency distribution of $\rho_{E}$ is characterized by a Lorentzian spectrum

$$
G(\omega)=\frac{1}{\pi} \frac{\delta \omega}{\delta \omega^{2}+\left(\omega-\omega_{0}\right)^{2}} .
$$

We have $\omega_{0}=2 \pi c /(914 \mathrm{~nm})$, and $1 / \delta \omega=9.703 \pm 0.124$ ps. The function $G(\omega)$ is normalized and has its support to a good approximation in the positive domain since $\omega_{0} \gg \delta \omega>0$,

$$
\int_{-\infty}^{\infty} G(\omega) \simeq \int_{0}^{\infty} G(\omega)=1
$$

(S42)
Its Fourier transform is given by

$$
\int_{0}^{\infty} G(\omega) e^{ \pm i \omega t} d \omega=e^{ \pm i \omega_{0} t} C(t)
$$

with

$$
C(t)=e^{-\delta \omega|t|}
$$

With this, the local trace distance, Eq. (S39), reduces to

$$
\Delta(\tau)=d\left|e^{-\delta \omega|t+\tau|}-e^{-\delta \omega|t-\tau|}\right|,
$$

which for $d=1 / 2$ yields Eq. (9) of the main manuscript. 